\title{
High Accuracy EBSD - A Review of Recent Applications, Innovations, and Remaining Challenges
}

\author{
Angus J. Wilkinson*, T Ben Britton*, Jun Jiang*, Graham Meaden** and David J. Dingley*** \\ * Department of Materials, University of Oxford, Parks Road, Oxford OX1 3PH, UK. \\ ** BLG Productions, 3 Sydenham Road, Bristol, BS6 5SH, UK. \\ *** Department of Physics, University of Bristol, Bristol, UK.
}

The implementation of cross-correlation analysis of EBSD patterns has allowed much improved angular sensitivity measurements to be achieved including the ability to determine the variations in elastic strains [1,2]. Cross-correlation functions between tests patterns and a reference pattern are calculated at selected sub-regions distributed across the patterns. A simple geometric analysis then allows the elastic strains and lattice rotations to be calculated from the shifts measured at these subregions. Sub-pixel resolution on the pattern shifts, typically to \pm 0.05 or better, leads to strain and rotation sensitivity of $\sim \pm 10^{-4}$.

In many situations a convenient location on the sample can be used to generate a reference pattern for which the strain state is well known, and in most cases zero. This is the case when studying the flow fields and residual stresses near indents in single crystals. Various wedge and pyramidal indents in representative $\mathrm{BCC}$ [3], diamond cubic [4] and HCP [5] crystals have been examined. FIG 1 shows the compressive radial and tensile hoop strains found around a Berkovich indent in Ti. Vaudin et al's comparison of EBSD with micro-Raman measurements shows good convergence as sampling depths are brought made more similar [4]. Analysis of deformation fields near crack tips and particles are important situations where strains fall to negligible levels in the far field so that good reference patterns can be obtained from regions well away from the crack or particle $[2,6,7]$.

In many more situations, such as a plastically deformed polycrystal, there is no obvious position on the sample from which to obtain a reference pattern corresponding to a known strain state. Fixing the absolute level of the strains in these situations remains the most significant challenge in continuing the development of the technique. One approach is to combine the local strain variations measured using the EBSD method with modeling at a longer length scale. A uniform strain state is then added to the measured EBSD strain variations to bring the average values into agreement with the model [6]. If characterizing the resulting deformation sub-structure is the main aim of the work then Nye's dislocation tensor analysis can be used to estimate the geometrically necessary dislocation (GND) density. This requires only gradients in the elastic strain and lattice rotation fields and so avoids the problem of assigning the absolute strain values. In all metal deformation studies we have made plastic flow results in rotations that are markedly larger than the elastic strains which can then be ignored when estimating the GND content. Indeed the lattice rotations can be sufficiently large so as to cause the cross-correlation analysis to give incorrect pattern shifts for parts of the pattern which change too much. We have recently implemented a robust fitting algorithm which identifies regions of the pattern for which the pattern shifts are suspect and down-weights their influence in an iterative scheme to determine the strain and rotation from the pattern shifts. This allows fuller coverage of the EBSD map as illustrated in FIG 2 (b) and (c) for a Cu sample deformed to $10 \%$ in tension.

The use of simulations to generate the reference patterns is an obvious approach to solving the 'reference pattern problem' and has been explored by several groups [8, 9]. The simulations must have sufficient fidelity and while crude kinematic methods are unacceptable, dynamical simulation show promise $[8,9,10]$. Aberrations in the EBSD detector optics can lead to significant artifacts in 
the measured strains [9] and so need to be determined and accounted for using methods given for example by Day [11]. However, the remaining barrier to successfully using simulations is the limited accuracy of currently available methods for determining the camera geometry. Errors in pattern centre position and specimen to screen distance are typically $\sim 0.5 \%$ of the screen width (or $\sim 5$ pixels) and this has been shown $[12,8,9]$ to induce errors of order $10^{-3}$ which are generally too large. Iterative fitting, camera movement, and shadow casting methods are all under investigation as possible means of providing the required sub-pixel accuracy in the camera geometry.

[1] A.J. Wilkinson, G. Meaden and D.J. Dingley, Ultramicroscopy, 106 (2006) 307-313.

[2] A.J. Wilkinson, G. Meaden and D.J. Dingley, Mater. Sci. Tech, 22 (2006) 1271-1278.

[3] A.J. Wilkinson and D. Randman, Philosophical Magazine, 90 (2010) 1159-1177.

[4] M.D. Vaudin et al Appl. Phys. Lett. 93 (2008) 193116.

[5] T.B. Britton, et al, Proc Royal Society A, 466 (2010) 695-719.

[6] P.S. Karamched and, A.J. Wilkinson Acta Materialia, 59 (2011), 263-272.

[7] J.D. Murphy et al IOP Conf Series Mater Sci \& Eng 3 (2009) 0120115

[8] J. Kacher et al Ultramicroscopy, 109 1148-1156.

[9] T.B. Britton et al Ultramicroscopy, 110 (2010) 1443-1453.

[10] A. Winkelmann et al Ultramicroscopy, 107 (2007) 414-421.

[11] A.P. Day, Journal of Microscopy 230 (2008) 472-486.

[12] S. Villert et al Journal of Microscopy 233 (2009) 290-301.
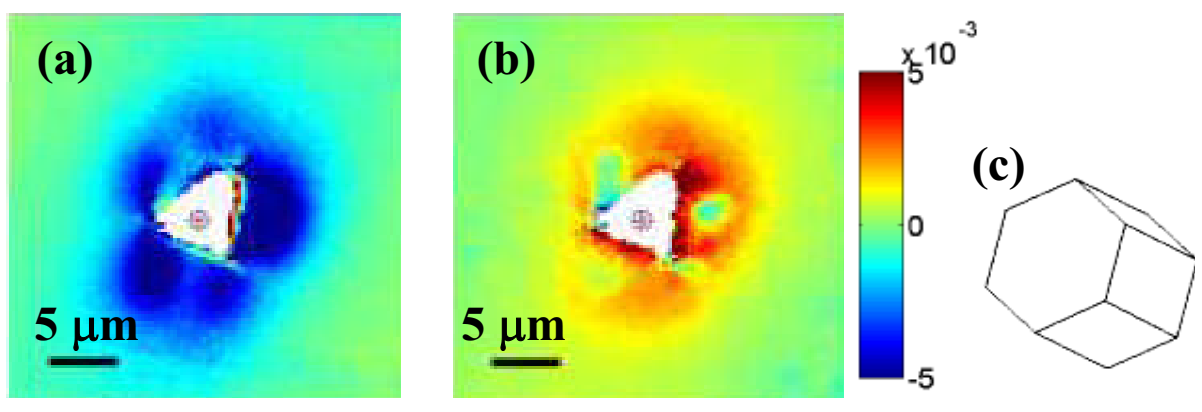

FIG. 1. (a) radial, and (b) hoop elastic strains near an indent in a commercially pure $\mathrm{Ti}$ crystal with orientation indicated in (c).
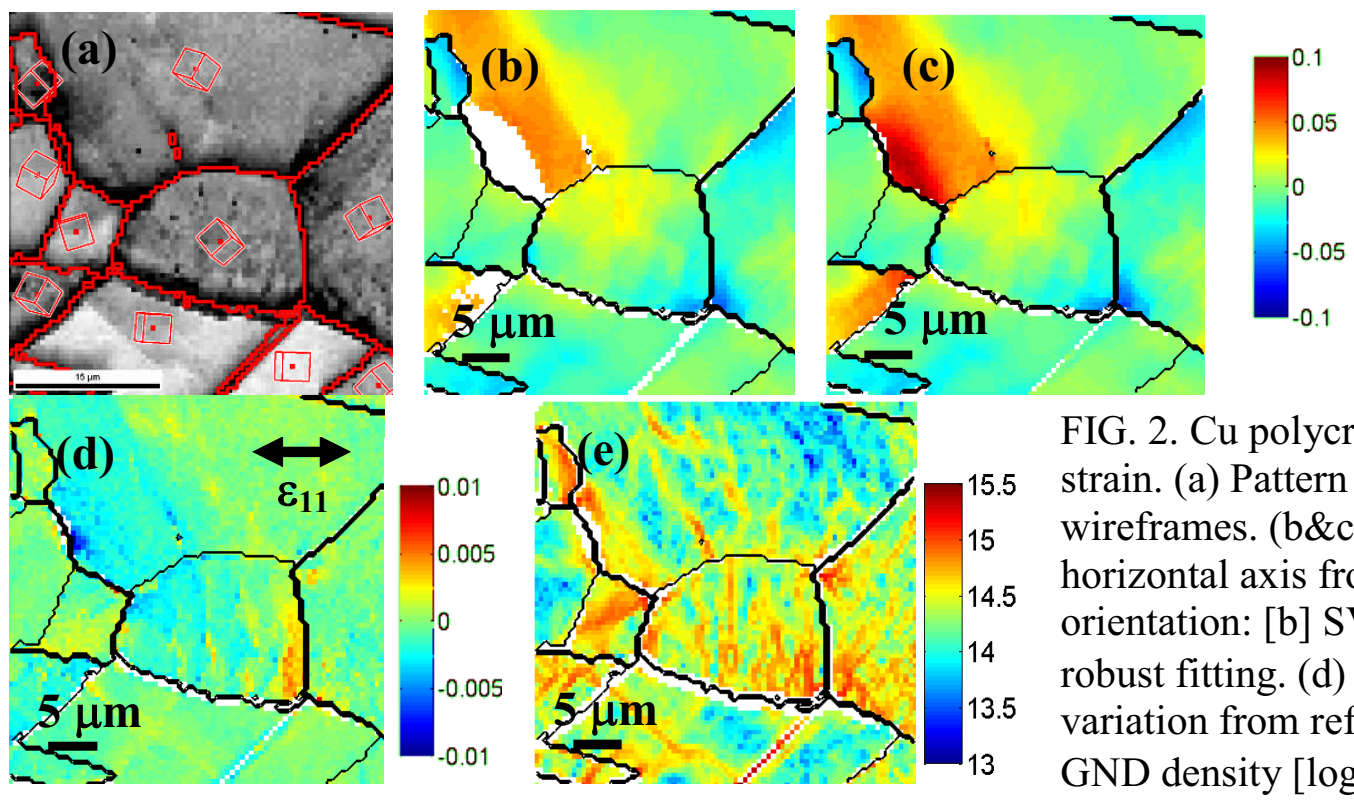

FIG. 2. Cu polycrystal 10\% tensile

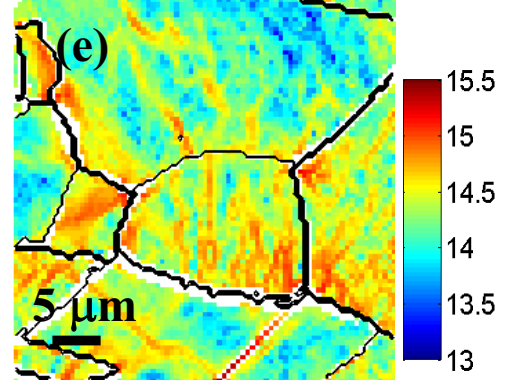

strain. (a) Pattern quality with crystal wireframes. (b\&c) Rotation about horizontal axis from reference orientation: [b] SVD solution and [c] robust fitting. (d) $\varepsilon_{11}$ elastic strain variation from reference value. (e) GND density $\left[\log \left(\rho\right.\right.$ in lines $\left.\left./ \mathrm{m}^{2}\right)\right]$. 